VIE SCIENTIFIQUE

\title{
BICENTENAIRE DE LA NAISSANCE D'AUGUSTE COMTE COLLOQUE INTERNATIONAL, MONTPELLIER ET PARIS, 11-16 MAI 1998
}

Auguste Comte (Montpellier, 19 janvier 1798 - Paris, 5 septembre 1857), promoteur de la «philosophie positive » et fondateur du «positivisme », a construit une des grandes philosophies systématiques du $\mathrm{XIX}^{\mathrm{e}}$ siècle. C'est une philosophie des sciences, qui réordonne toute l'encyclopédie et y ajoute la « sociologie ». C'est aussi une philosophie politique et sociale qui veut rien moins que réorganiser la société, en France, en Occident et dans le monde. Et c'est une philosophie qui s'est déployée en une nouvelle religion : la "religion de l'Humanité ».

La philosophie d'Auguste Comte semble maintenant relever d'un passé révolu. Et le succès du positivisme au $\mathrm{XIX}^{\mathrm{e}}$ siècle s'est soldé par sa désaffection au $\mathrm{xx}^{\mathrm{e}}$. Cette œuvre puissamment systématique a cependant eu une importance décisive pour de nombreux auteurs, ne serait-ce que pour s'y opposer. Les colloques du Bicentenaire se proposent de replacer cette entreprise dans son temps, tout en essayant de réévaluer son intérêt pour la réflexion d'aujourd'hui.

Le Colloque international se déroulera en deux parties:

$1^{\mathrm{re}}$ partie, Montpellier, 11-13 mai 1998 : «Le temps d'Auguste Comte " - Palais des congrès "Corum ", salon du Belvédère;

$2^{e}$ partie, Paris, 14-16 mai 1998 : « Auguste Comte. Science et politique » - Bibliothèque nationale de France, auditorium Colbert.

\section{Renseignements}

Association pour le Bicentenaire d'Auguste Comte 10 rue Monsieur-le-Prince, 75006 , Paris

Tél. 0144414668 - Télécopie : 0144414671

et Annie Petit, professeur à l'université Paul-Valéry, Route de Mende, BP 5043, 34032 Montpellier Cedex 1

Tél. 0467142478 - Télécopie : 0467142265 